\title{
The Vestibular System Mediates Sensation of Low-Frequency Sounds in Mice
}

\author{
Gareth P. Jones ${ }^{1}$, Victoria A. Lukashkina ${ }^{1}$, IAn J. Russell ${ }^{1}$, and Andrei N. Lukashkin ${ }^{1}$ \\ ${ }^{1}$ School of Life Sciences, University of Sussex, Falmer, Brighton, BN1 9QG, UK
}

Received: 22 September 2009; Accepted: 5 August 2010; Online publication: 4 September 2010

\begin{abstract}
The mammalian inner ear contains sense organs responsible for detecting sound, gravity and linear acceleration, and angular acceleration. Of these organs, the cochlea is involved in hearing, while the sacculus and utriculus serve to detect linear acceleration. Recent evidence from birds and mammals, including humans, has shown that the sacculus, a hearing organ in many lower vertebrates, has retained some of its ancestral acoustic sensitivity. Here we provide not only more evidence for the retained acoustic sensitivity of the sacculus, but we also found that acoustic stimulation of the sacculus has behavioral significance in mammals. We show that the amplitude of an elicited auditory startle response is greater when the startle stimuli are presented simultaneously with a low-frequency masker, including masker tones that are outside the sensitivity range of the cochlea. Masker-enhanced auditory startle responses were also observed in otoconia-absent Nox3 mice, which lack otoconia but have no obvious cochlea pathology. However, masker enhancement was not observed in otoconia-absent Nox3 mice if the low-frequency masker tones were outside the sensitivity range of the cochlea. This last observation confirms that otoconial organs, most likely the sacculus, contribute to behavioral responses to lowfrequency sounds in mice.
\end{abstract}

Keywords: cochlea, vestibular, sacculus, Nox3 mouse, auditory startle, auditory masker

Correspondence to: Ian J. Russell · School of Life Sciences · University of Sussex·Falmer, Brighton, BN1 9QG, UK. email: i.j.russell@ sussex.ac.uk

\section{INTRODUCTION}

Mammals have a remarkable sense of hearing, with auditory ranges specifically adapted to their particular acoustic niche. This sensitivity is achieved mainly by the cochlea, a relatively new evolutionary adaptation compared with the much more ancient vestibular system. Recent studies have demonstrated, however, that parts of the mammalian vestibular system, notably the sacculus, historically one of the first acoustically sensitive organs (Popper et al. 1982), have retained the ability to detect acoustic stimuli previously associated only with the cochlea. There is further evidence that this stimulation may have behavioral significance (Todd 2001). Acoustic sensitivity of the sacculus in mammals might be expected as consequences of both its evolutionary history and anatomical location. In mice, for example, the sacculus is located behind the oval window membrane, directly in the line of vibration caused by movement of the stapes. It is clearly visible through the oval window once the stapes have been removed.

The vestibular systems of birds and mammals including cats (McCue and Guinan 1994, 1995), monkeys (Young et al. 1977), guinea pigs (Cazals et al. 1983; Didier and Cazals 1989), and pigeons (Wit et al. 1984) have been found to respond to acoustic stimuli, often with good evidence that the vestibular afferents innervating the sacculus are the most acoustically sensitive. Evidence from primates indicates that afferent projections from the sacculus reach the medial vestibular nucleus or inferior vestibular nucleus, which then projects bilaterally to the spinal cord (Stein and Carpenter 1967). In humans, for example, this pathway mediates vestibular-evoked myogenic potentials 
(VEMPs), in which acoustic excitation of the vestibular system causes twitches in various muscles, most obviously the cervical muscles (Bickford et al. 1964; Ferber-Viart et al. 1999). Although the typical stimuli used are also within the human auditory range, an auditory origin of the VEMPS is ruled out by the fact that they still occur in patients with hearing loss (Sheykholeslami and Kaga 2002; Wang and Young 2003; Murofushi et al. 2005) .

With its ability to respond to acoustical stimulation, the vestibular system has the potential to extend the hearing range of mammals and hence their ability to detect low frequency sounds of behavioral significance. For example, the frequency range of the mouse cochlea, defined by sensitivity to sound stimulation below $80 \mathrm{~dB}$ SPL, is approximately $4-75 \mathrm{kHz}$, although this varies depending on age and mouse strain (Nyby 2001). There are three areas of peak sensitivity that coincide with behaviorally relevant frequency ranges; the area of best sensitivity at the lower range is around $10-18 \mathrm{kHz}$ and at ultrasonic frequencies, $40 \mathrm{kHz}$ and $70 \pm 10 \mathrm{kHz}$ (Nyby 2001; Müller et al. 2005).

In this article, we investigate whether mice can respond to low-frequency sounds. We demonstrate that low-frequency pure tones, which have stimulus parameters outside the sensitivity range of the mouse cochlea (Taberner and Liberman 2005; Müller et al. 2005), can facilitate acoustic startle responses (ASRs) caused by a high-frequency tone that is within the range of the frequency and sensitivity of the cochlea (Fig. 1). This facilitation disappears in vestibular deficient mice proving that the vestibular system in mice can mediate the detection of low-frequency sounds.

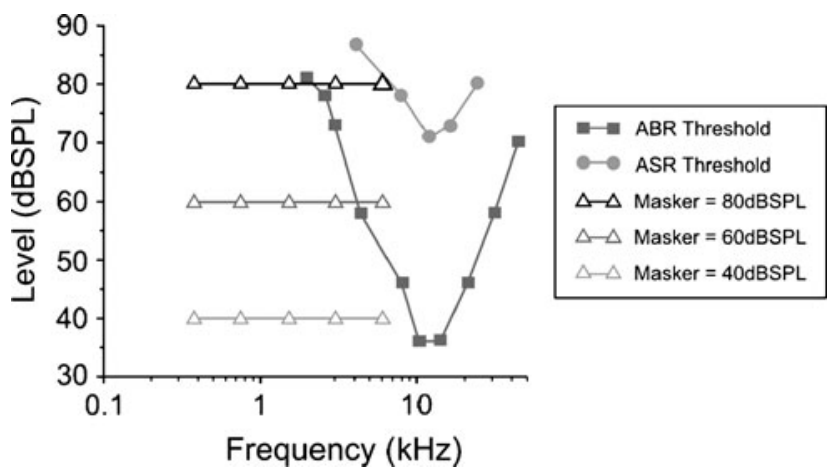

FIG. 1. ASR (circles; age 1 month) and ABR (squares) thresholds for CBA mice compared with the levels and frequencies used as background maskers in the experiments (triangles). Only the higher levels (60 and $80 \mathrm{~dB} \mathrm{SPL}$ ) at the higher frequencies ( 3 and $6 \mathrm{kHz}$ ) are above $A B R$ threshold. ASR threshold curve was modified from Parham and Willott (1988). ABR threshold curve was modified from Müller et al. (2005).

\section{METHODS}

The ASR is a short latency motor reflex in response to loud, abrupt sounds, common to most mammals. It is often used as a behavioral method to assess hearing in mice; ASR protocols are most revealing when a startle eliciting stimulus (probe) is used in conjunction with additional stimuli (maskers). The ASR can be modified by maskers presented simultaneously or preceding the startle stimulus. For example, Carlson and Willott (2001) employed simultaneous masker tones of different frequencies in conjunction with a probe stimulus to reveal facilitation of the elicited ASR (Carlson and Willott 2001). However, masker frequencies below the auditory range of the mouse cochlea have not been included in previous studies. The experiments described in this article test the hypothesis that low-frequency pure tones are detected by mice and are capable of modifying the ASR in a similar fashion to audible background tones.

In total, two strains of mice were used; wild-type $\mathrm{CBA} / \mathrm{CaJ}$ maintained at the University of Sussex and Nox3 C57BL6/J mice originally obtained from The Jackson Laboratory and then maintained and outbred to a CBA/CaJ background at the University of Sussex. All mice were housed individually for at least a week prior to experimentation and were used between 6 and 8 weeks old to avoid any complications caused by age-related hearing changes. The Nox3 mice carry a series of recessive mutations that knock out the NADPH oxidase 3 gene preventing the development of the otoconia in the sacculus and utricle in homozygous mutants with consequent vestibular deficits (henceforth referred to as otoconia-absent Nox3 mice) (Paffenholz et al. 2004). The ASR from CBA/ CaJ mice was initially measured to provide baseline data because this particular mouse strain demonstrated a larger ASR amplitude. For the sake of clarity, results for the CBA/CaJ mice are presented separately to the otoconia-present Nox3 mice as the absolute size of their ASRs varied slightly due to a small difference in weight between the litters of each type used.

All experiments were conducted in an acoustic booth (IAC, UK). Pure tones for sound stimulation and white noise for sound calibration were generated digitally, converted to analogue by a DT3010/32 (Data Translation) data acquisition card at a rate of $200 \mathrm{kHz}$ and presented through a pair of loudspeakers, one for the startle eliciting "probe" tone (2.5" Motorola piezo tweeter) and one for the background "masker" tone ( 3 " Eurotec $8 \Omega$ loudspeaker). Sound pressure was monitored using a Bruel \& Kjaer 4133 1/4-inch microphone. The sound system was calibrated free field in situ for frequencies between 100 and 70,000 kHz. Known sound pressure levels were expressed in $\mathrm{dB}$ SPL re: $2 \times 10^{-5} \mathrm{~Pa}$. Startle 
responses were recorded via an analogue accelerometer (laboratory designed and constructed) and digitized by the same data acquisition card at a rate of $200 \mathrm{kHz}$. The experimental chamber was arranged with both loudspeakers in front of the mouse to ensure binaural stimulation. The wire mesh cage was mounted on the accelerometer and was small enough to restrict the mouse's movements, which would otherwise diminish the ASR amplitude (Fig. 2a). Stimuli were presented only during periods in which the mouse was settled, as determined from video observation and accelerometer output.

\section{A}

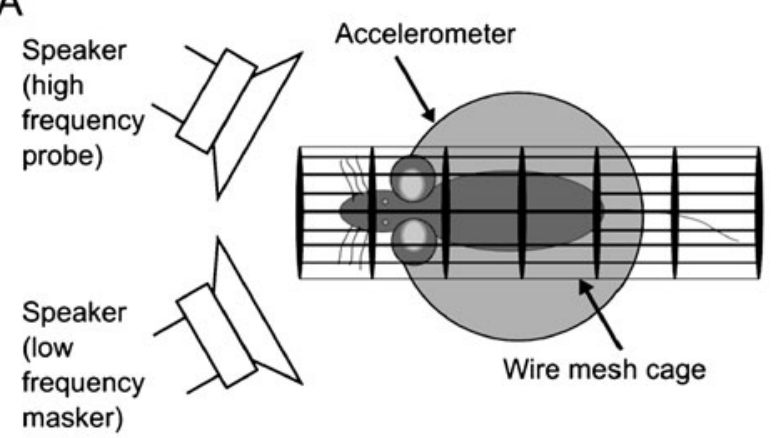

B

Probe Only

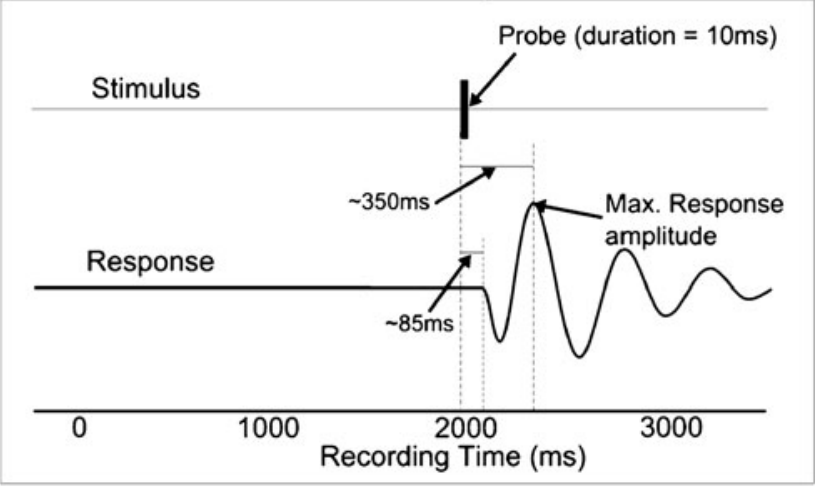

C Probe and Masker

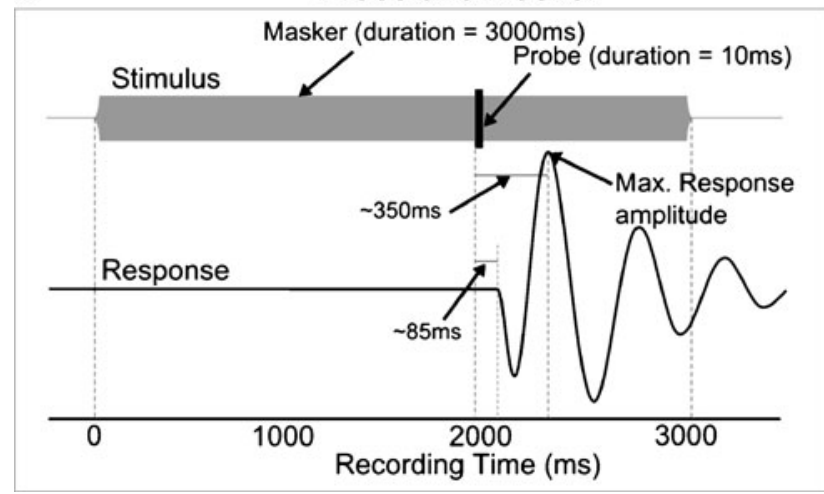

FIG. 2. A The startle chamber schematic. B The temporal order of probe alone presentations. C The temporal order of probe and masker presentations (C). The onset of the masker preceded that of the probe by $2,000 \mathrm{~ms}$ and continued for a total of 3,000 ms.
Each experiment involved manipulating two variables; masker frequency and probe level, with the masker level kept constant. The probe frequency was determined prior to starting the experiments by finding the most salient startle eliciting frequency. For both the CBA and the Nox3/CBA mice, this was $14 \mathrm{kHz}$ and was not changed over the course of the experiments. Suitable probe levels for eliciting the ASR were also determined prior to starting the experiments; for the CBA, mice probe levels of 80 and $90 \mathrm{~dB}$ SPL were used and for the Nox3/CBA mice, $90 \mathrm{~dB}$ SPL (80 dB SPL was not used for the Nox3/CBA mice as it did not elicit a large enough ASR). Individual mice were subject to three experiments; one for each masker level (40, 60, and $80 \mathrm{~dB}$ SPL), separated by at least $24 \mathrm{~h}$. Each possible combination of stimulus conditions was presented to the mouse in randomized blocks. For example, for the CBA mice, the block size was 12-containing all possible combinations of two different probe levels and six different masker conditions (Table 1). These blocks of presentations were repeated until a total of 120 presentations (10 for each masker frequency) had been performed (or a 1-hour time limit was reached). The "masker off" conditions were used as a baseline against which masked responses for the same probe level were normalized.

The presentation order of each individual probe/ masker combination is shown in Figure $2 b$ (without masker) and c (with masker). The onset of the masker always preceded that of the probe by $2 \mathrm{~s}$ and was on for a total of $3 \mathrm{~s}$. The probe stimulus had a rise/fall time of $2 \mathrm{~ms}$ to increase its startle saliency, whereas the masker tone had a longer $10 \mathrm{~ms}$ rise/fall time in order to avoid it potentially eliciting a startle at its onset at the higher levels.

ASRs were normalized by dividing the ASR to the combination of probe tone and masker $\left(P_{\mathrm{M}}\right)$ by the ASR to the probe tone alone $\left(P_{0}\right)$,. In Figures 3, 5, 6, and 7 , the normalized data are expressed as the "Startle Ratio" $\left(P_{\mathrm{M}} / P_{0}\right)$. After normalization, multiway repeated-measures analyses of variance (ANOVAs) were performed with startle ratio as the dependent variable on the data as presented in Figures 3, 5, 6, and 7. Post hoc two-way $t$-tests were then performed on each masker frequency/level combination against the normalized response to the probe on its own to indicate which masker conditions significantly facilitated the ASR amplitude. These comparisons were judged significant at $p=0.01$ (Bonferroni correction of $p=0.05$ for five multiple comparisons) and are indicated on the graphs with astrexes. Data and statistical analysis were performed in Matlab R2006b (The MathWorks, Inc.) and Origin 7 (OriginLab Corporation).

All Nox3/CBA mice used were bred from heterozygous parents and were phenotyped after completion 
TABLE 1

The 12 possible combinations of stimulus conditions when using two probe levels and six masker frequencies (including no masker)

\begin{tabular}{lllllll}
\hline $\begin{array}{l}\text { Probe level } \\
(d B S P L)\end{array}$ & \multicolumn{9}{l}{ Masker frequency $(\mathrm{kHz})$} \\
\hline 80 & Masker off & 0.375 & 0.75 & 1.5 & 3 & 6 \\
90 & Masker off & 0.375 & 0.75 & 1.5 & 3 & 6 \\
\hline
\end{tabular}

Each block of 12 was randomized before presentation

of the experiments. Attempts to use behavioral assays to identify the otoconia-absent Nox3 mice proved unreliable, and as such, all experiments using the mice were conducted blind. The homozygous mutants were identified postmortem, after completing all experiments by inner ear dissection. The presence (or lack) of otoconia in the sacculus was checked visually via the oval window after removal of the stapes. Out of a total of 38 dissections from four separate litters, 10 otoconia-absent mice were identified, 6 of which were prior used in the ASR experiments. The numbers of mice for each group used in the behavioral experiments are 8, 4, and 4 for CBA mice, otoconia-present mice, and otoconia-absent mice, respectively. The numbers of otoconia-present mice used for the compound action potential (CAP) and distortion product otoacoustic emission (DPOAE) experiments are 5 and 9, respectively. The corresponding numbers for otoconia-absent mice are 3 and 3 (see Fig. 4).

To measure DPOAEs and CAPs of the auditory nerve, mice were anesthetized with ketamine $(0.12 \mathrm{mg} / \mathrm{g}$ body weight i.p.) and xylazine $(0.01 \mathrm{mg} /$ g body weight i.p.) for DPOAE procedures or with urethane (ethyl carbamate, $2 \mathrm{mg} / \mathrm{g}$ body weight, i.p.) for CAP procedures. The animals were tracheotomized, and their core temperature was maintained at $38^{\circ} \mathrm{C}$. To measure CAPs, a caudal opening was made in the ventrolateral aspect of the right bulla to reveal the round window. CAPs were measured from the round window membrane using glass pipettes filled with artificial perilymph with tip diameters of 50$100 \mu \mathrm{m}$ (recording bandwidth $>30 \mathrm{kHz}$ ). Signals were amplified with a recording bandwidth of $\mathrm{DC}-$ $100 \mathrm{kHz}$.

To measure DPOAEs and CAPs, sound was delivered via a probe with its tip within $1 \mathrm{~mm}$ of the tympanic membrane and coupled to a closed acoustic system comprising two MicroTech Gefell 1-inch MK102 microphones for delivering tones and a Bruel \& Kjaer 3135 $\frac{1}{4}$-inch microphone for monitoring sound pressure at the tympanum. The sound system was calibrated in situ for frequencies between 1 and $70 \mathrm{kHz}$ using a measuring amplifier, and known SPLs were expressed in $\mathrm{dB}$ SPL re $2 \times 10^{-5} \mathrm{~Pa}$. White noise and tone pulses with rise-fall times of $0.2 \mathrm{~ms}$ were synthesized by a Data Translation 3010 data acquisition board, attenuated, and used for sound system calibration and the measurement of electrical and acoustical cochlea responses. To measure DPOAEs, the levels of the $\mathrm{f} 2$ tone were set $10 \mathrm{~dB}$ SPL below that of the $\mathrm{f} 1$ tone, with the frequency ratio of $\mathrm{f} 2 / \mathrm{fl}$ set to 1.23. DPOAE threshold curves were constructed from measurements of the level of the $\mathrm{f} 2$ tone that produced a $2 \mathrm{f1}-\mathrm{f} 2$ DPOAE with a level of $0 \mathrm{~dB}$ SPL. System distortion during DPOAE measurements was $80 \mathrm{~dB}$ below the primary tone levels. On the basis of laser diode interferometer measurements from the spiral lamina and bony wall of the mouse cochlea (Legan et al. 2000; unpublished data), only sound pressure and not linear acceleration was delivered to the cochlea at levels and

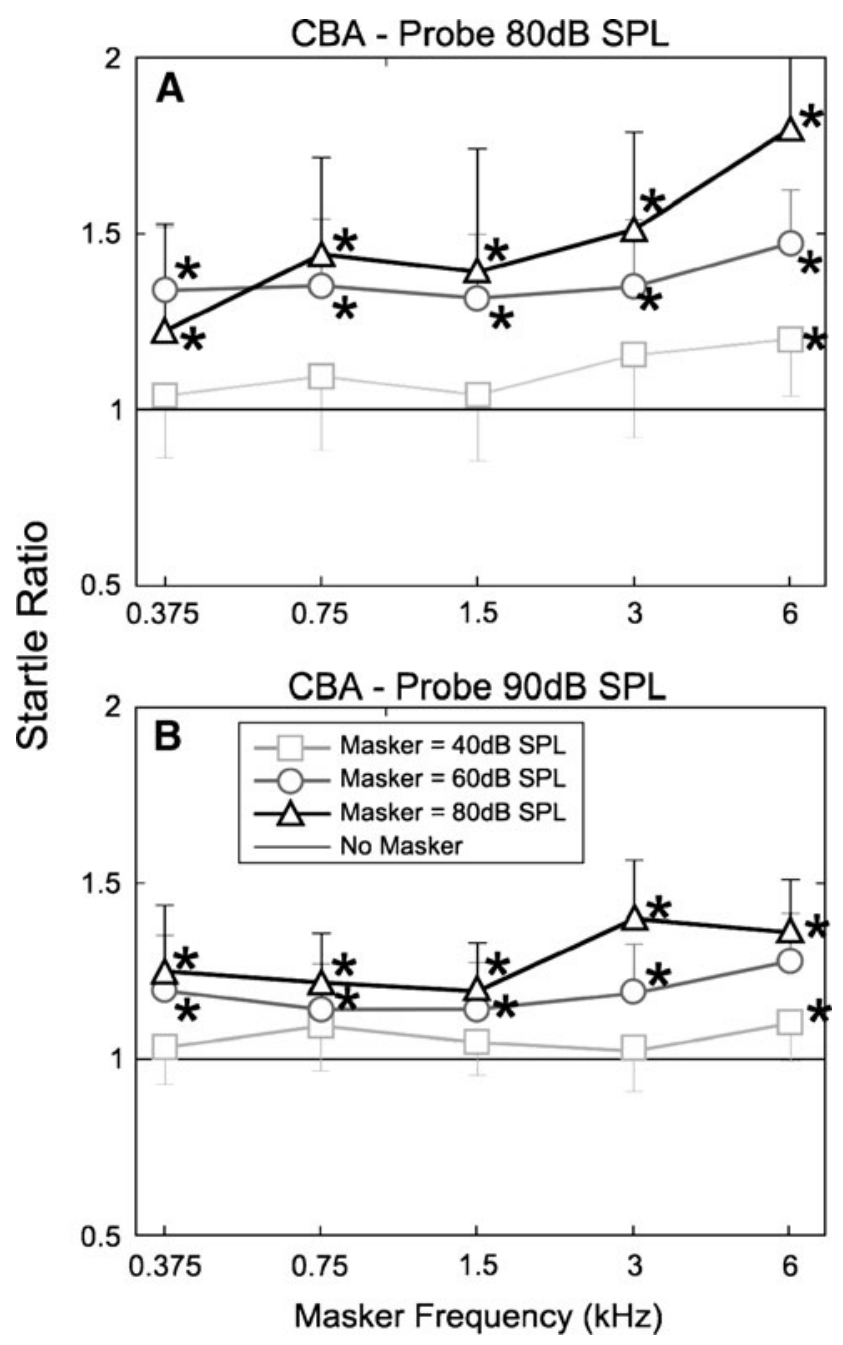

FIG. 3. Startle ratio (startle amplitude, compared with that due to the probe tone on its own; $y=1$ ) as a function of masker frequency for CBA mice. A $80-\mathrm{dB}$ SPL, $14-\mathrm{kHz}$ probe. B $90-\mathrm{dB}$ SPL, $14-\mathrm{kHz}$ probe. In this and all subsequent figures, startle ratio is the ASR due to the combination of probe tone and masker $\left(P_{\mathrm{M}}\right)$ divided by the ASR to the probe tone alone $\left(P_{0}\right)\left(P_{\mathrm{M}} / P_{0}\right)$. Error bars indicate standard deviation; * indicates significance at $p \leq 0.0, n=7$, for 40 - and $60-\mathrm{dB}$ SPL points for both probes and $n=8$ for all $80-\mathrm{dB}$ SPL maskers. 


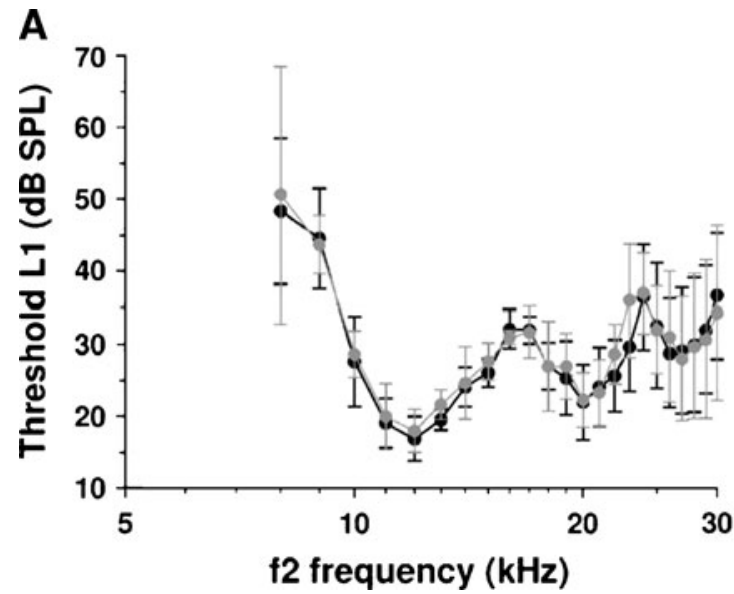

FIG. 4. Threshold levels of acoustical responses (A) recorded in nine otoconia-present (black) and three otoconia-absent (gray) mice and neural responses (B) recorded in five otoconia-present (black) and three otoconia-absent (gray) mice. A Threshold level L1 (mean \pm SD) of the

frequencies within the range of the sound system used in these experiments.

All procedures involving animals were performed in accordance with UK Home Office regulations with approval from the local ethics committee.

\section{RESULTS}

Multiway repeated-measures ANOVA of the ASRs recorded from the CBA mice (Fig. 3) with startle ratio as the dependent variable and probe level, masker level, and masker frequency as factors showed significant effects of probe level $(p<0.001, F=30.6, d f=$ $1)$, masker frequency $(p<0.001, F=92.6 d f=5)$, and masker level $(p<0.001, F=47.1 d f=2)$. Interactions were also observed between masker frequency and masker level $(p<0.001, F=11.7, d f=10)$; ASRs recorded in the presence of masker tones increased in amplitude with masker level and frequency (Fig 3). Data points shown to be significantly different (two-way $t$ test) to the probe only baseline are marked with an asterisk ( $p=0.01$ or better). These maskers included frequencies within and below the range of the mouse cochlea (Fig. 1). Facilitation in the presence of masker frequencies, which are below the frequency range and sensitivity of the cochlea (masker frequencies $1.5,0.75$, and $0.375 \mathrm{kHz}$ ), caused facilitation when presented at levels of $60 \mathrm{~dB}$ SPL and above. No significant facilitation was observed for any of the masker frequencies when presented at the lowest masker level (40 dB SPL), except for at $6 \mathrm{kHz}$.

Cochlear function in homozygous Nox3 mutant mice which lack otoconia is not affected (Paffenholz et al. 2004). This observation was confirmed in our study through the measurement of similar DPOAE

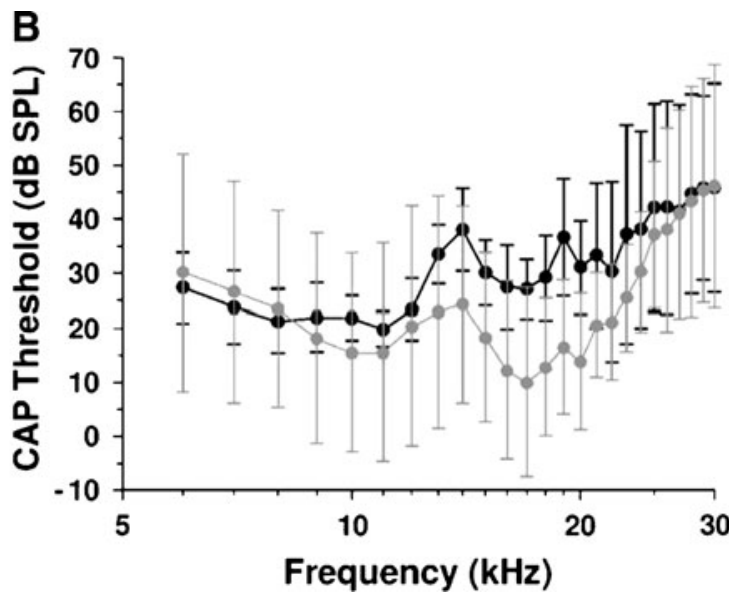

low-frequency primary $\mathrm{f} 1$ required to generate $0-\mathrm{dB}$ SPL DPOAE at frequency $2 \mathrm{f} 1-\mathrm{f} 2$ for different frequencies of the high-frequency primary f2. B CAP threshold levels (mean \pm SD) for pure-tone stimulation. Number of animals $n$ is indicated within the figure panels.

and CAP audiograms from Nox3 mice regardless of the presence or absence of otoconia in the macula of the sacculus (Fig. 4).

The ASRs recorded from the otoconia-present littermates of the otoconia-absent Nox3 mice exhibited similar facilitation to the CBA mice (Fig. 5), whereas this facilitation differed for the otoconia-absent mice (Fig. 6). Multiway repeatedmeasures ANOVA with startle ratio as the dependent variable and phenotype, masker frequency, and masker level as factors showed significant effects of phenotype $(p<0.001 F=40.7, d f=1)$, masker frequency $(p<0.001 F=14.3, d f=4)$, and masker level $(p<0.001 \quad F=37.1, d f=2)$. Interactions occurred between phenotype and masker frequency $(p<$ $0.001, F=5.27, d f=4)$ and between masker frequency

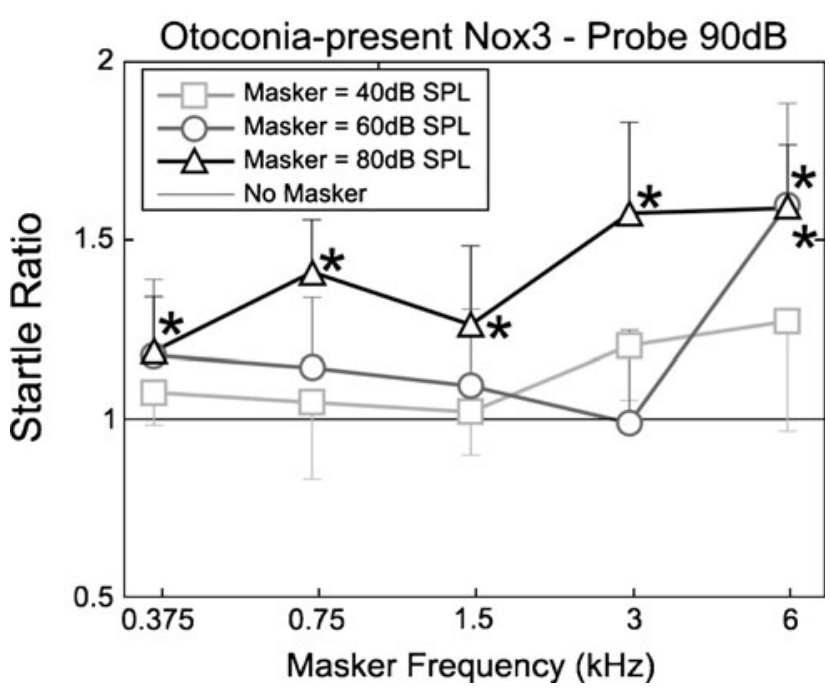

FIG. 5. Startle ratio (startle amplitude, compared with that due to the probe tone on its own; $y=1$ ) as a function of masker frequency for otoconia-present Nox 3 mice at the masker levels shown. $n=4$ for all points. 


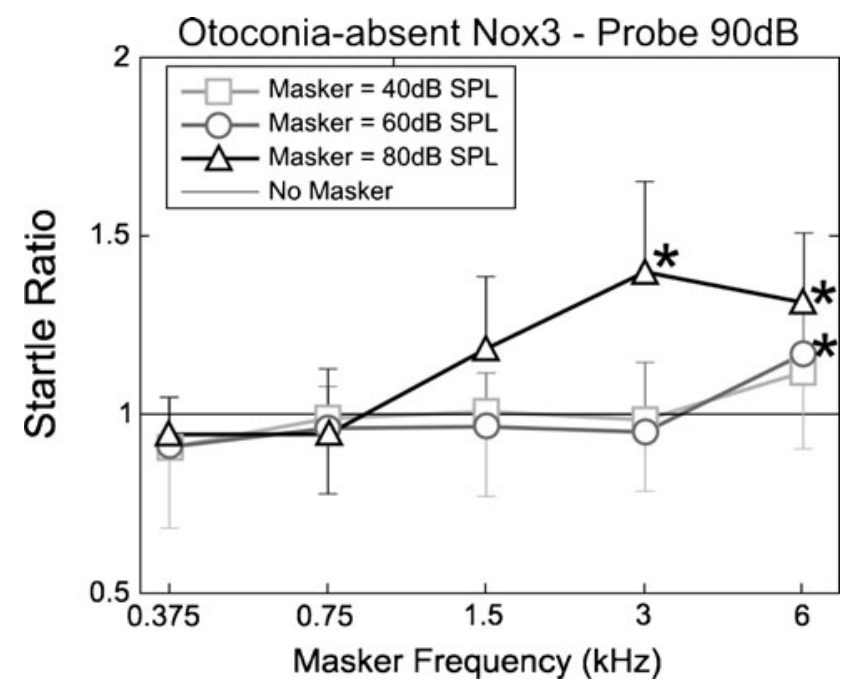

FIG. 6. Startle ratio (startle amplitude, compared with that due to the probe tone on its own; $y=1$ ), as a function of masker frequency for otoconia-absent Nox3 mice at the masker levels shown. $n=4$ for all points.

and masker level $(p<0.001, F=4.14, d f=8)$. As with the CBA mice, the ASR of otoconia present mice was facilitated for all masker frequencies when presented at $80 \mathrm{~dB}$ SPL. Facilitation at lower masker levels (40 and $60 \mathrm{~dB}$ SPL) occurred only at $6 \mathrm{kHz}$ (Fig. 5). This facilitation was again generally greater for higher frequencies ( 3 and $6 \mathrm{kHz}$ ) but still present for the masker frequencies below the mouse's auditory range (1.5, 0.75, and $0.375 \mathrm{kHz})$. Again, facilitation increased with masker level for all frequencies, although with a higher threshold; between 60 and $80 \mathrm{~dB}$ SPL compared with between 40 and $60 \mathrm{~dB}$ SPL for the CBA mice. The ASRs of otoconia-absent Nox3 mice were facilitated only by masker frequencies well within the auditory range of the mouse cochlea, at frequencies $3 \mathrm{kHz}$ (at $80 \mathrm{~dB}$ SPL) and $6 \mathrm{kHz}$ (at $60 \mathrm{~dB}$ SPL and above) (Fig. 6). No significant facilitation was observed for the lower frequency maskers at any level.

Figure 7 shows a comparison of the 80-dB masker levels for each group of mice. Multiway ANOVA of this data with startle ratio as the dependent variable and phenotype and masker frequency as factors indicates significant effects of phenotype $(p<0.001, F=11.0, d f=$ $2)$, masker frequency $(p<0.001, F=61.5, d f=5)$, and interaction between phenotype and masker frequency $(p<0.001, F=4.86, d f=10)$.

\section{DISCUSSION}

ASRs measured in CBA and otoconia-present Nox3 mice were facilitated at all masker frequencies that were tested. The maskers at 3 and $6 \mathrm{kHz}$ are within the frequency range of the cochlea and were deliv- ered at levels within its range of sensitivity. It is highly likely, therefore, that the facilitation of the ASRs at these frequencies was mediated via the cochlea and auditory nerve. Effective facilitation of the ASR was also achieved with maskers at frequencies $(0.375,0.75$, and $1.5 \mathrm{kHz}$ ) and levels (60 and $80 \mathrm{~dB}$ SPL) outside the frequency-sensitivity range of the mouse cochlea, as measured from threshold, single unit, auditory nerve data (Taberner and Liberman 2005), and auditory brainstem responses (Fig. 1). The facilitation must, therefore, be a result of input from another sensory modality that is also excited, albeit indirectly, by sound pressure (see "Methods").

In contrast to the CBA and otoconia-present Nox3 mice, ASRs recorded from the otoconia-absent Nox3 mice were not facilitated by masker frequencies below $3 \mathrm{kHz}$ (Fig. 7), even though DPOAE and CAP audiograms from otoconia-present or otoconia-absent Nox3 mice are indistinguishable (Fig. 4). The Nox3 mutation is reported to affect only the otoconia of the vestibular system (Paffenholz et al. 2004). In agreement with this observation, our dissections of the peripheral auditory system did not reveal any other abnormalities in the gross structure of the inner ear at the macroscopic level, with the cochlea and vestibular apparatus appearing to be similar in both otoconiapresent and otoconia-absent Nox3 mice. The ASR thresholds were very similar for all three groups of mice, although the ASR amplitude was slightly smaller for the Nox3 mice, due to individuals in these litters of mice being slightly smaller in size than the mice in the CBA litters.

On the basis of our observations that tones with frequencies below $3 \mathrm{kHz}$ facilitate ASRs caused by audible tones in otoconia-present Nox3 mice but not

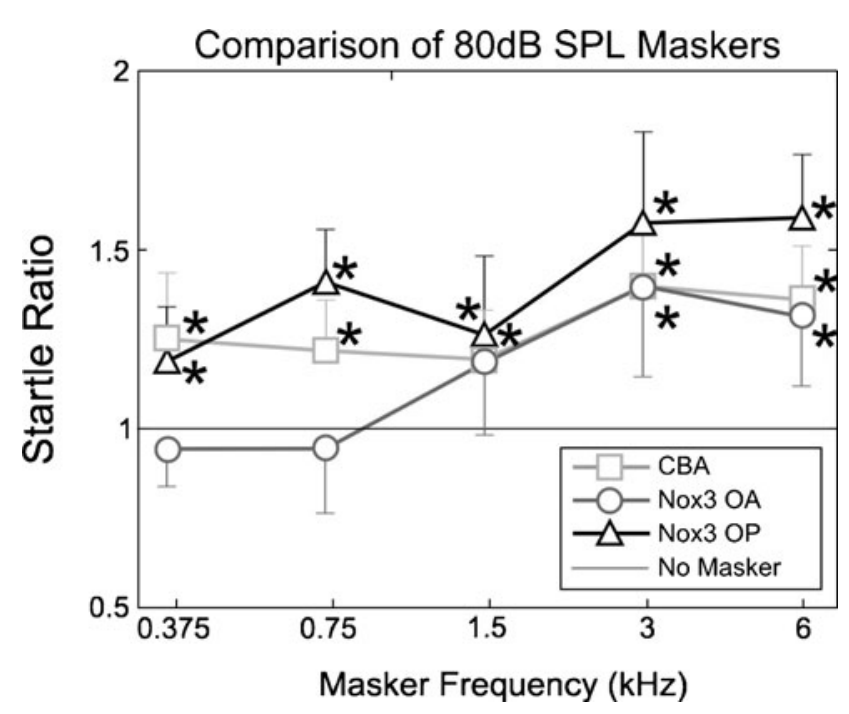

FIG. 7. Startle ratio (startle amplitude, compared with that due to the probe tone on its own; $y=1$ ), as a function of masker frequency at constant 80-dB SPL level for CBA, otoconia-present (OP) Nox3, and otoconia absent $(\mathrm{OA})$ Nox3 mice. 
in otoconia-absent Nox3 mice, where the otoconia is missing from the gravitational receptors, we propose that at least two systems are implicated in the ASR facilitation. The cochlea mediates facilitation with the masker tones above $3 \mathrm{kHz}$ and the vestibular system (most likely the sacculus) mediates facilitation for masker tones with frequencies below $3 \mathrm{kHz}$. This proposal is also supported by the finding that one of the two peaks observed in VEMPs is of vestibular origin with the other of cochlea origin (Ferber-Viart et al. 1999). Cross-modal modification of the ASR by the sacculus/utricle has been described previously using free fall rather than acoustic stimulation of the vestibular system (with the facilitation being greatest when the stimulation of each modality was temporally synchronized relative to the point of summation) (Yeomans et al. 2002).

The neuronal circuitry underlying the ASR is thought to involve the caudal pontine reticular nucleus (PnC) innervating motor neurons and receiving input from brainstem nuclei including the dorsal cochlea nucleus (DCN), cochlea root nucleus (CRN), medial cochlea nucleus (MCN), and lateral superior olive (LSO) (Koch 1999). Of these innervating nuclei, the CRN pathway has the shortest latency and depolarizes cells in the PnC to close to firing threshold. It is hypothesized that any further input from the other nuclei will then elicit a startle response (Koch 1999). It has been shown that the sacculus has projections reaching the DCN (Burian and Gstoettner 1988), providing a possible mechanism by which it can facilitate the ASR. Perhaps a more parsimonious explanation for the augmentation of the acoustically activated ASR through acoustic activation of the vestibular system is that the vestibular signals activate the limbic system; i.e., rumbling, low-frequency, vibrations cause anxiety, thereby eliciting a stronger startle response. Support for this latter suggestion has yet to be forthcoming because, for example, Schanbacher et al. (1996) discovered that lesions of the amygdala do not affect the enhancement of the ASR by background noise. These proposed mechanisms also predict that, as observed in the data presented in this article, vestibular stimulation by acoustic stimuli is not necessarily capable of eliciting an ASR independently of acoustic stimuli within the range of the cochlea (at least not by the same auditory pathway). Acoustic stimulation of the vestibular system can, however, contribute to a concurrent ASR elicited by a cochlear pathway (which activates the PnC).

The data in this article support and extend previous studies showing the retained acoustic sensitivity of the mammalian sacculus and for the first time demonstrates how this detection has a physiologically significant effect on an important behavioral reflex.

\section{ACKNOWLEDGEMENTS}

We thank an anonymous reviewer for their constructive comments and help and Mr. James Hartley for technical support. G.J. was supported by a research studentship from the BBSRC. Research was supported by the MRC.

\section{REFERENCES}

Bickford RG, Jacobson JL, Cody DTR (1964) Nature of average evoked potentials to sound and other stimuli in man. Ann NY Acad Sci 112:204-218

Burian M, Gstoettner W (1988) Projection of primary vestibular afferent fibres to the cochlear nucleus in the guinea pig. Neurosci Lett 84:13-17

Carlson S, Willott JF (2001) Modulation of the Acoustic Startle Response by background sound in C57BL/6J mice. In: Willott JF (ed) The handbook of mouse auditory research from behaviour to molecular biology. CRC Press LLC, pp 82-90

Cazals Y, Aran JM, Erre JP, Gullhaume A, Aurousseau C (1983) Vestibular acoustic reception in the guinea pig: a saccular function? Acta Otolaryngol 95:211-217

Didier A, Cazals Y (1989) Acoustic responses recorded from the saccular bundle on the eighth nerve of the guinea pig. Hear Res $37: 123-128$

Ferber-Viart C, Dubreuil C, Duclaux R (1999) Vestibular evoked myogenic potentials in humans a review. Acta Otolaryngol 119:6-15

Koch M (1999) The neurobiology of startle. Prog Neurobio 59:107128

Legan KP, Lukashikina VA, Goodyear RJ, Kossl M, Russell IJ, Richardson GP (2000) A targeted deletion in a-tectorin reveals that the tectorial membrane is required for the gain and timing of cochlear feedback. Neuron 28:273-285

Mccue MP, Guinan JJ JR (1994) Acoustically responsive fibers in the vestibular nerve of the cat. J Neurosci 14:6058-6070

Mccue MP, Guinan JJ JR (1995) Spontaneous activity and frequency selectivity of acoustically responsive vestibular afferents in the cat. J Neurophysiol 74:1563-1572

Müller M, Hünerbein K, Hoidis S, SMolders J (2005) A physiological place-frequency map of the cochlea. Hear Res 202:63-73

Murofushi T, Iwasaki S, Taka Y, Takegoshi H (2005) Sound-evoked neurogenic responses with short latency: are they of vestibular origin? Clin Neurophysiol 116:401-405

NyBY JG (2001) Auditory communication among adults. In: Willott JF (ed) The handbook of mouse auditory research from behaviour to molecular biology. CRC Press LLC, pp 3-18

Paffenholz R, Bergstrom R, Pasutto F, Wabnitz P, Munroe R, Jagla W, Heinzmann U, Marquardt A, Bareiss A, Laufs J, Russ A, Stumm G, Schimenti J, Bergstrom D (2004) Vestibular defects in head-tilt mice result from mutations in Nox3, encoding an NADPH oxidase. Genes Dev 18:486-491

Parham K, Willott J (1988) Acoustic startle response in young and aging C57BL/6J and CBA/J mice. Behav Neurosci 102:881-886

Popper A, Platt C, Saidel W (1982) Acoustic function in the fish ear. Trends Neurosci 5:276-280

Schanbacher A, Koch M, Pilz PK, Schnitzler HU (1996) Lesions of the amygdala do not affect the enhancement of the acoustic startle response by background noise. Physiol Behav 60:13411346

SheYkholeslami K, Kaga K (2002) The otolithic organ as a receptor of vestibular hearing revealed by vestibular-evoked myogenic potentials in patients with inner ear anomalies. Hear Res 165:62-67 
Stein B, Carpenter M (1967) Central projections of portions of the vestibular ganglia innervating specific parts of the labyrinth in the rhesus monkey. Am J Anat 120:281-317

Taberner AM, Liberman MC (2005) Response properties of single auditory nerve fibres in the mouse. J Neurophysiol 93:557-569

Todd N (2001) Evidence for a behavioral significance of saccular acoustic sensitivity in humans. J Acoust Soc Am 110:380-390

WANG S, Young Y (2003) Vestibular evoked myogenic potentials using simultaneous binaural acoustic stimulation. Hear Res 185:43-48
Wit H, Bleeker J, Mulder H (1984) Responses of pigeon vestibular nerve fibers to sound and vibration with audio frequencies. J Acoust Soc Am 75:202-208

Yeomans J, Li L, Scott B, Frankland P (2002) Tactile, acoustic and vestibular systems sum to elicit the startle reflex. Neurosci Biobehav Rev 26:1-11

Young E, Fernández C, Goldberg J (1977) Responses of squirrel monkey vestibular neurons to audio-frequency sound and head vibration. Acta Otolaryngol 84:352-360 